\title{
DEVELOPMENT OF THE MULTI-ANGLE STRATOSPHERIC AEROSOL RADIOMETER (MASTAR)
}

\author{
Matthew DeLand $^{1}$, Peter Colarco ${ }^{2}$, Matthew Kowalewski $^{3}$, Nick Gorkavyi $^{1}$, Luis Ramos-Izquierdo ${ }^{2}$ \\ ${ }^{1}$ Science Systems and Applications, Inc. (SSAI) \\ ${ }^{2}$ NASA Goddard Space Flight Center (GSFC) \\ ${ }^{3}$ GESTAR/Universities Space Research Association (USRA)
}

\begin{abstract}
The contribution of atmospheric aerosols to the Earth's energy budget is an important and relatively uncertain component of the Earth system, with correspondingly large uncertainties implied for climate models. Comprehensive observations of the vertical profile of aerosol extinction are needed to accurately characterize these particles (e.g. composition, size, spatial and temporal distribution) to properly account for their climate impacts and constrain models. Satellite limb scattering measurements provide superior results for observations of stratospheric aerosols compared to occultation measurements. We are developing a compact instrument called Multi-Angle Stratospheric Aerosol Radiometer (MASTAR) that simplifies the current OMPS Limb Profiler design to fit into a $3 \mathrm{U}$ Cubesat configuration, and also provides important supplemental information through the use of multiple simultaneous viewing directions.
\end{abstract}

Index Terms - Stratospheric aerosol, limb scattering, Cubesat

\section{INTRODUCTION}

Previous assessments of aerosol-climate effects have focused on the impact of anthropogenic aerosols in the troposphere. For example, a recent major report clearly states "...clouds and aerosols continue to contribute the largest uncertainty to estimates and interpretations of the Earth's changing energy budget" [1]. However, there is a growing body of evidence suggesting the additional importance of aerosols in the upper troposphere and lower stratosphere (UT/LS). Recent studies indicate a positive trend in stratospheric aerosol loading since 2002, despite a lack of increased input to the stratosphere from any major volcanic eruptions [2]. The long-term persistence of stratospheric aerosols over months to years, and their resulting radiative effects, impact the ability to understand climate variations on multiple time scales.

Proper characterization of these aerosol inputs requires altitude-resolved measurements of aerosol extinction as a key geophysical variable to initialize transport calculations. Extensive spatial sampling and good vertical resolution are needed to capture the inhomogeneous vertical and horizontal distribution of stratospheric aerosols. Integrated measurements such as stratospheric aerosol optical depth (sAOD) represent a valuable constraint on profile observations, but do not provide the additional information about vertical distribution that is needed for proper modeling of transport effects and radiative forcing estimation.

Multiple observing methods (e.g. occultation, lidar, limb scattering) can be used to monitor stratospheric aerosols. Solar occultation measurements determine extinction directly, but only sample a single latitude each day with either sunrise or sunset events. Space-based lidar measurements provide better sampling and high vertical resolution, but produce a different measured parameter (backscatter coefficient) and have signal-to-noise limitations for stratospheric purposes. Limb scatter measurements provide comprehensive spatial and temporal sampling, but must characterize the aerosol size distribution (or phase function) in order to retrieve extinction values. Of these options, limb scattering provides perhaps the most useful combination of vertical resolution, spatial and temporal sampling, and data quality. 


\section{CURRENT MEASUREMENTS}

The Ozone Mapping and Profiling Suite (OMPS) Limb Profiler (LP) instrument on the Suomi NPP (SNPP) satellite currently collects limb scattering data to create an aerosol extinction data record, which begins in 2012. The LP instrument provides stratospheric aerosol profiles with $\sim 1.8 \mathrm{~km}$ vertical resolution and global coverage [3]. However, the sensitivity of this instrument to aerosols has a geographic dependence, because its viewing geometry (looking backwards along the orbit track) gives high scattering angles (and thus lower sensitivity to aerosols) in the Southern Hemisphere. LP measurements at low scattering angles in the Northern Hemisphere can be a factor of 10-30 more sensitive to aerosols that $\mathrm{SH}$ measurements. The Sun-synchronous orbit of S-NPP also limits the LP sampling to a single local time, with a multi-day gap between revisit times at any location.

Our objective is to supplement the S-NPP OMPS LP measurements by developing a simplified, compact instrument configuration focused on stratospheric aerosol observations. The Multi-Angle Stratospheric Aerosol Radiometer (MASTAR) design also utilizes the limb scattering technique, but adds multiple simultaneous viewing directions to increase spatial sampling (Figure 1) and reduce hemispheric phase function sampling sensitivity.

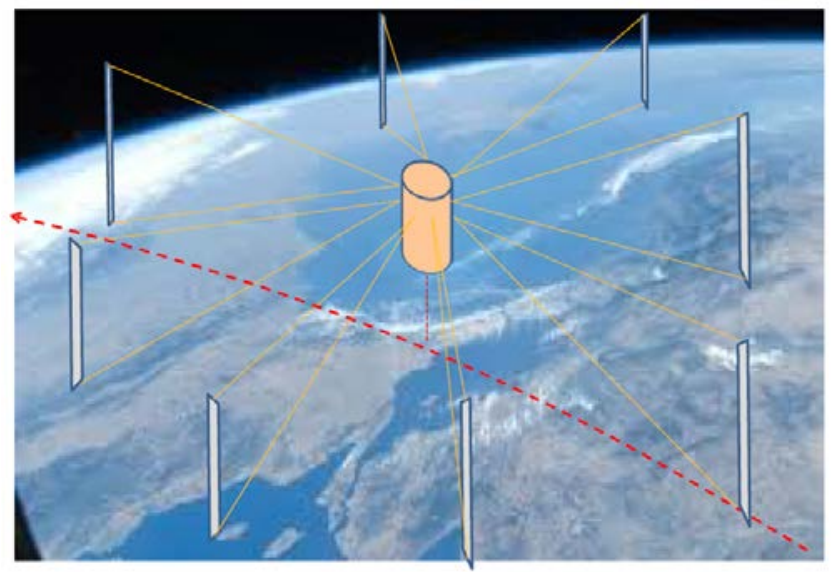

Figure 1. The MASTAR instrument will view the Earth's limb with eight apertures simultaneously. Each slit measures a radiance profile over the altitude range $0-60 \mathrm{~km}$.

The viewing geometry of the MASTAR instrument ensures that locations along the satellite orbit track will be sampled with both forward and backward scattering view angles within approximately 15 minutes throughout the orbit. This approach gives a more balanced distribution of sensitivity to aerosols at all latitudes in both hemispheres. The complementary measurements collected by MASTAR at multiple scattering angles will also provide substantial statistical leverage to constrain the phase function (and thus particle size distribution) that characterizes aerosols at a given location.

\section{INSTRUMENT DESIGN}

In order to facilitate rapid development and possible deployment, we have simplified the LP design for implementation in a $3 \mathrm{U}$ Cubesat configuration. Atmospheric light enters the instrument horizontally through eight apertures at the top of the spacecraft, then is directed downward to a detector using an octagonal prism (Figure 2). Lenses in front of each prism create a longer effective focal length than would otherwise be possible with the Cubesat frame. Reimaging optical elements below the prism are used to create focused images on the CCD detector.

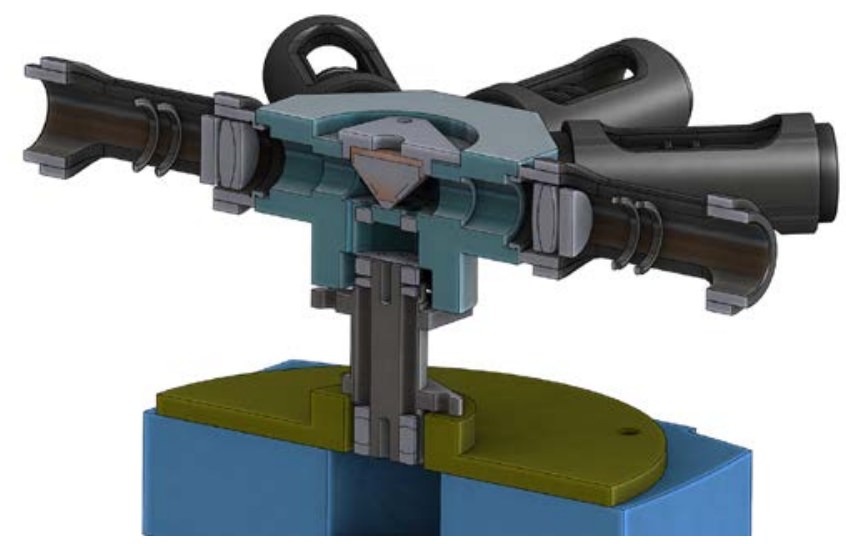

Figure 2. Side view (cutaway) of the MASTAR optical design. Light enters from the side into each baffle tube, passes through lenses, then is redirected by the central prism downward to the $\mathrm{CCD}$ detector.

The projection of each aperture onto a CCD using a narrow slit provides a radiance profile with $0.5 \mathrm{~km}$ vertical resolution between $0-60 \mathrm{~km}$. The slit images place the brightest portion of each profile (looking close to the Earth's surface) towards the outer portion of the CCD. This feature maximizes the separation between neighboring slit images in order to mitigate stray light contamination. 
Wavelength selection is accomplished using simple bandpass filters. In this design, 6 of the 8 apertures are dedicated to aerosol measurements (Figure 3). The primary wavelength $(670 \mathrm{~nm})$ is chosen for heritage with the current OMPS LP V1.0 aerosol extinction retrieval algorithm [4]. Additional sampling at $850 \mathrm{~nm}$, a wavelength that is less subject to saturation from Rayleigh scattering, extends the effective altitude coverage further down into the UT/LS.

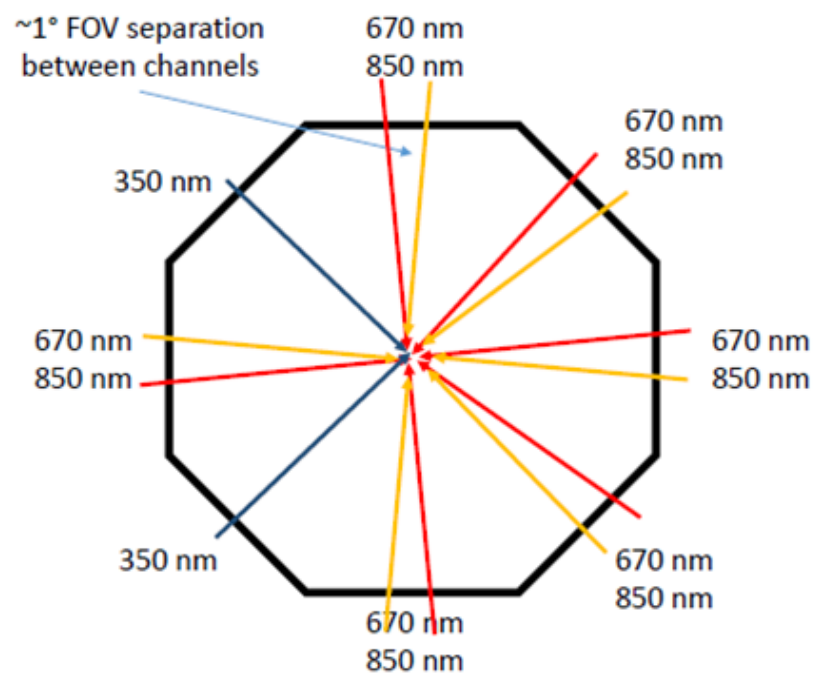

MASTAR Octagon

Figure 3. Schematic view of the MASTAR measurement system from above, showing the assignment of wavelength choices to each viewing direction.

Since accurate altitude registration is critically important for limb scattering measurements, two apertures make measurements at $350 \mathrm{~nm}$ in orthogonal directions to supplement the onboard attitude control system. These radiance profiles can be used to evaluate satellite pointing by applying the Rayleigh scattering attitude sensor (RSAS) approach [5].

\section{STATUS}

Initial development of a laboratory prototype instrument for this project was supported by NASA GSFC internal funding. Current MASTAR development is supported by a NASA Instrument Incubator Program (IIP) grant. Optical and mechanical design has been completed, and all primary components have been procured. Figure 4 shows the octagonal central prism.

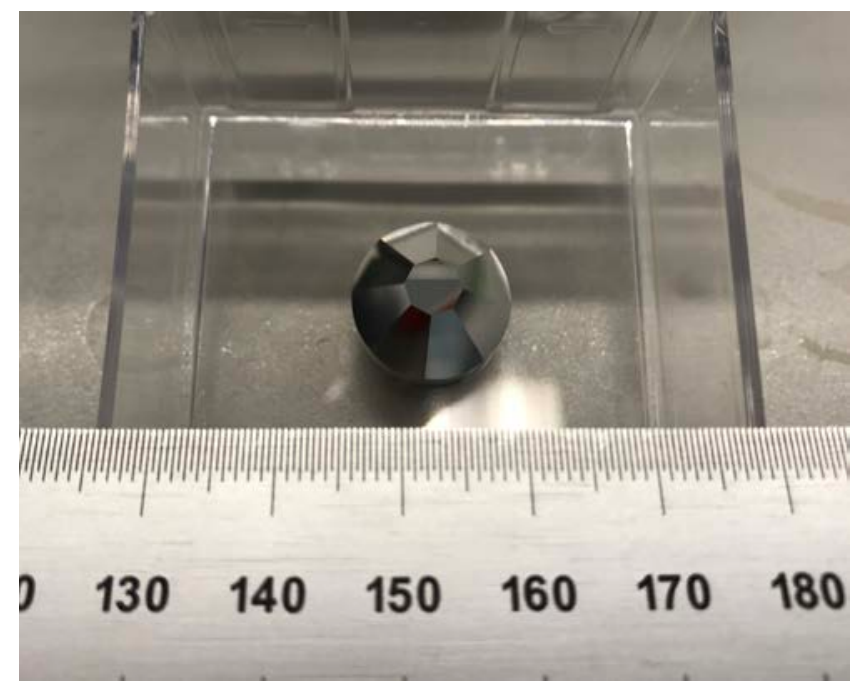

Figure 4. Fabricated central prism for the MASTAR instrument. The prism diameter is approximately $12 \mathrm{~mm}$.

Integration of the central optics (spectral filters, slit plate, reimaging optics) is in progress at this time (May 2018). This process requires a precise sequence of steps because of the placement of many optical elements inside the overall instrument. Figure 5 shows the central prism assembly and field lens assembly prior to final integration.

We anticipate conducting laboratory tests of MASTAR with an integrating sphere source in May 2018, followed by outdoor field tests. Further developments will include evaluation of nominal onorbit thermal variations (including occasional direct solar views into an aperture) on instrument performance, and development of a modified optical design that incorporates the slit plate field stops with the focal plane array. Both tasks are designed to advance the MASTAR design towards flight-ready capability. 


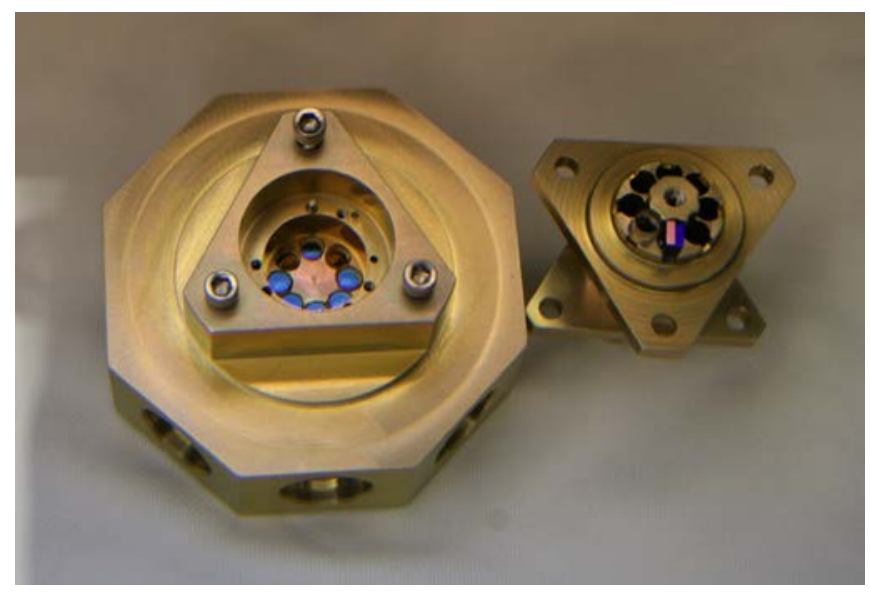

Figure 5. The central prism assembly (left) and field lens assembly (right) of the MASTAR instrument. Both assemblies are shown inverted relative to their final orientation in the integrated instrument. Reimaging optics for each of the 8 apertures are visible as small circles in the center of each component.

\section{ACKNOWLEDGEMENTS}

Funding support for this project was provided by NASA grants NNX17AD43G and 80NSSC18K0076, and by NASA Goddard Space Flight Center Code 610AT.

\section{REFERENCES}

[1] IPCC, Climate Change 2013: The Physical Science Basis. Contribution of Working Group I to the Fifth Assessment Report of the Intergovernmental Panel on Climate Change, T. F. Stocker et al. (eds.), Cambridge Univ. Press, Cambridge, UK, 1535 pp, 2013.

[2] S. Kremser, et al., "Stratospheric aerosol - Observations, processes, and impact on climate", Rev. Geophys., 54, Wiley, doi:10.1002/2015RG000511, 2016.

[3] G. Jaross, P. K. Bhartia, G. Chen, M. Kowitt, M. Haken, Z. Chen, P. Xu, J. Warner, and T. Kelly, "OMPS Limb Profiler instrument performance assessment", J. Geophys. Res. Atmos., 119, doi:10.1002/2013JD020482, AGU Publ., 2013.

[4] R. Loughman, P. K. Bhartia, Z. Chen, P. Xu, E. Nyaku, and G. Taha, "The Ozone Mapping and Profiler Suite (OMPS) Limb Profiler (LP) Version 1 aerosol extinction retrieval algorithm: Theoretical basis", Atmos. Meas. Tech. Discuss., https://doi.org/10.5194/amt-2017-299, Copernicus Publ., 2017.

[5] L. Moy, P. K. Bhartia, G. Jaross, R. Loughman, N. Kramarova, Z. Chen, G. Taha, G. Chen, and P. Xu, "Altitude registration of limb-scattered radiation", Atmos. Meas. Tech., 10, Copernicus Publ., p. 167-178, 2017. 